Pacific Journal of Mathematics

SOLVABILITY OF INVARIANT DIFFERENTIAL OPERATORS 


\section{SOLVABILITY OF INVARIANT DIFFERENTIAL OPERATORS ON METABELIAN GROUPS}

\section{Peter OHRing}

In this work we use non-commutative harmonic analysis in the study of differential operators on a certain class of solvable Lie groups. A left invariant differential (a differential operator that commutes with left translations on the group) can be synthesized in terms of differential operators on lower dimensional spaces. This synthesis is easily described for a certain class of simply connected solvable Lie groups, those arising as semi-direct products of simply connected abelian groups.

We derive sufficient conditions for the semiglobal solvability of left invariant differential operators on such groups in terms of the lower dimensional differential operators. These conditions are seen to be satisfied for certain classes of second order differential operators, thus yielding semiglobal solvability. Specifically elliptic, sub-elliptic, transversally elliptic and parabolic operators are investigated.

1. Introduction. In the mid 1970's the study of differential operators with polynomial coefficients that arise as invariant operators on nilpotent Lie groups began. Group representation theoretic criteria for hypo-ellipticity and solvability of such operators were found. Rothschild [11] gave such criteria for the local solvability of homogeneous left invariant differential operators on the Heisenberg group based on some ideas of Rockland [10]. These ideas were extended to all simply connected nilpotent Lie groups by Corwin in [1]. In recent years these ideas and methods have been extended to type I solvable Lie groups. Lipsman [6] gave criteria for local solvability of left invariant differential operators on type I solvable Lie groups, thus extending the horizon of study to differential operators with more general (transcendental) coefficients.

In the work done so far on one sided invariant operators, noncommutative harmonic analysis plays an important role. Specifically the Plancherel theory is an essential ingredient. In this work we demonstrate an alternative approach, one which doesn't use the Plancherel theory. One advantage is that we obtain solvability results for operators on non-type I groups for which there is no reasonable Plancherel theory. In addition our approach is successful in obtaining 
results on type I groups for which the Plancherel theory approach is not.

Let $L$ be a left invariant differential operator on a solvable Lie group $G$ where $G$ is the semi-direct product of 2 abelian groups $S$ and $N$, where $\operatorname{dim} S=1$ and $N$ is normal in $G$. $L$ can be thought of as a linear operator on $L^{2}(G)$ with $D(L)=C_{c}^{\infty}(G)$.

We can put $L$ in block form by taking the partial Euclidean Fourier transform over $N$. Each block is a differential operator acting on a copy of $L^{2}(\mathbb{R})$. This is analogous to the diagonalization of a constant coefficient differential operator on $\mathbb{R}^{n}$.

In group representation theoretic terms we decompose the right regular representation of $G$ as a direct integral of representations induced from the normal subgroup $N$. This decomposition yields a decomposition of $L$ into a direct integral of differential operators, $\pi^{\xi}(L), \xi \in \hat{N}$ acting on $L^{2}(\mathbb{R}) .(\hat{N}=$ dual of $\hat{N}=$ characters on $N$.)

The underlying philosophy here is to give criteria for the solvability of $L$ in terms of the solvability of its Fourier transforms, the $\pi^{\xi}(L)$ 's. These operators are acting on a lower dimensional space and in theory should be easier to investigate. In practice there are many obstacles. The most natural way of proving solvability of the $\pi^{\xi}(L)$ 's is to show that they are boundedly invertible on $L^{2}(\mathbb{R})$. In Lipsman [6] there are many concrete examples of operators investigated in this manner, and in Theorem 3.2 we also give examples of such operators. For those operators whose Fourier transforms are not invertible on $L^{2}(\mathbb{R})$, alternative methods must be used. In Lipsman [7], Lipsman has relaxed his condition of bounded invertibility on $L^{2}(\mathbb{R})$ to bounded invertibility with respect to some Sobolev norm.

One of our approaches has been to use weighted Hilbert spaces. In the proof of Theorem (3.1), where we investigate sub-laplacians, we deduce the solvability of the $\pi^{\xi}(L)$ 's from the invertibility of certain associated operators, $A_{\xi}$, as operators from $L^{2}\left(\mathbb{R}^{2}, \omega_{1}\right)$ to $L^{2}\left(\mathbb{R}^{2}, \omega_{2}\right)$ where $\omega_{1}, \omega_{2}$ are appropriate weights on the plane. In $\S 4$ we examine operators whose Fourier transforms are first order ordinary differential operators and we deduce solvability by the classical method of introducing an integrating factor.

To solve $L u=f, f \in C_{c}^{\infty}(G)$, for $u$, we examine the associated equations $\pi^{\xi}(L) u_{\xi}=f_{\xi}$ where $f_{\xi}$ denotes the partial Fourier transform of $f$ over $N$. Though we may be able to solve this equation for $u_{\xi}$, $u_{\xi}$ may not be in $L^{2}(\mathbb{R})$. Worse yet we may not be able to invert the Fourier transform to obtain a solution $u$ to the original problem. 
Theorem 2.1 deals with these obstacles and gives criteria for semiglobal solvability. This is the main result of $\S 2$ which also contains some background material and definitions. (Throughout we adopt the notation used in Lipsman [6].)

In $\S \S 3$ and 4 we look at examples of certain classes of second order differential operators which are seen to satisfy the hypotheses of Theorem 2.1. Let $T$ be the generator of $S$, and let $X_{0}, \ldots, X_{n-1}$ be a basis for $N$. In $\S 3$ we investigate operators of the form $L=T^{2}+\sum_{i \in I} X_{i}^{2}$, $I \subseteq\{0, \ldots, n-1\}$ and $L_{X}=L+X, X \in n$. Semiglobal solvability of these operators is seen to follow from Theorem 2.1. Examples of differential operators with transcendental coefficients which arise as left invariant operators having the above form are given in $\S 3$.

In $\S 4$ we prove global solvability for certain "heat" operators as an application of Theorem 2.1. In the above notation these are operators of the form $L=T-\sum_{i \in I} X_{i}^{2}$.

2. Solvability criteria. In this section we will state and prove a theorem which gives sufficient conditions for solvability of left invariant operators on solvable Lie groups which arise as semi-direct products of simply connected abelian Lie groups. In later sections we will apply this theorem to prove solvability results of specific classes of second order operators. First we introduce some notation and quickly recount some of the background theory we shall need:

Let $G$ be a Lie group, with right Haar measure $d g$. We write $\mathbf{g}$ for the Lie algebra, $U(\mathbf{g})$ for the universal enveloping algebra and $Z(\mathbf{g})$ for its center. We identify $\mathbf{g}$ with the algebra of left invariant vector fields on $G$ by:

$$
X \in \mathbf{g} \Rightarrow(X f)(g)=\frac{d}{d t} f(g \cdot \exp t X)_{t=0}, \quad f \in C^{\infty}, g \in G .
$$

The element $X Y \in U(\mathbf{g}), X, Y \in g$, acts on $C^{\infty}(G)$ by $X Y(f)(g)=$ $X(Y f)(g)$. In this way $U(\mathbf{g})$ is identified with the algebra of left invariant operators on $G$.

If $\pi$ is a unitary representation of $G$, acting on a Hilbert space $H$, then it lifts to $g$ by the formula

$$
\pi(X) v=\frac{d}{d t}(\pi(\exp t X) v)_{t=0}, \quad X \in g, v \in C^{\infty}(\pi) .
$$

It follows that

$$
\pi[X, Y]=\pi(X) \pi(Y)=\pi(Y) \pi(X) .
$$

Thus $\pi$ extends naturally to $U(\mathbf{g})$. 
Let $\rho$ denote the right regular representation of $G$. $\rho$ acts on $L^{2}(G)$ and is given by the formula $\rho(g) f(h)=f(h g), g, h \in G, f \in L^{2}(G)$. For $X \in \mathbf{g}$ and $f \in C^{\infty}(G)$ we have

$$
\rho(X) f(h)=\frac{d}{d t}(\rho(\exp t X) f(h))_{t=0}=\frac{d}{d t} f(h \cdot \exp t X)_{t=0}=X f(h) .
$$

Thus we can identify $X \in \mathbf{g}$ with $\rho(X)$. This identification naturally extends to $U(\mathbf{g}) . L \in U(\mathbf{g})$ is identified with $\rho(L)$. This identification is useful in arriving at representation theoretic criteria for the solvability of $L$.

Now let $G$ be the semi-direct product of two simply connected abelian groups. Let $G=N \cdot S, N \approx \mathbb{R}^{n}, S \approx \mathbb{R}, N$ normal in $G$. Fix $T \in \mathbf{s}, T \neq 0$. The multiplication law for the group is given by

$$
(\exp X, \exp t T) \cdot(\exp Y, \exp s T)=\left(\exp \left(X+e^{\operatorname{ad}_{t T}} Y\right), \exp (s+t) T\right) .
$$

As $N$ is a vector group we can identify $\mathbf{n}^{*}$ with $\hat{N} . \xi \in \mathbf{n}^{*}$ is identified with the character defined by $\xi(\exp X)=e^{i\langle\xi, X\rangle}$ for $X \in \mathbf{n}$. For $\xi \in \mathbf{n}^{*}$ let $\pi^{\xi}$ denote $\operatorname{ind}_{N}^{G} \xi$. We can realize $\pi^{\xi}$ as acting on $L^{2}(\mathbb{R})$ :

(2.1) $\pi^{\xi}(\exp X, s) f(t)=e^{i\left\langle\xi, e^{\operatorname{ad}_{t} T} X\right\rangle} f(t+s)$,

$$
X \in \mathbf{n}, \xi \in \mathbf{n}^{*}, f \in L^{2}(\mathbb{R}) .
$$

Let $\rho^{\prime}$ denote the unitary representation of $G$ acting on $L^{2}\left(\mathbf{n}^{*} \cdot \mathbb{R}\right)$ defined by the direct integral

$$
\rho^{\prime}(g)=\int_{\mathbf{n}^{*}} \pi^{\xi}(g) d \xi
$$

If $\rho_{N}$ denotes the right regular representation of $N$, then $\rho_{N}(n) \sim$ $\int_{\mathbf{n}^{*}} \xi(n) d \xi$ where the equivalence is given by Fourier transform on the vector group $N$.

Now $\rho \sim \operatorname{ind}_{N}^{G} \rho_{N}$. Since inducing commutes with direct integrals we can conclude that $\rho(g) \sim \rho^{\prime}(g)=\int_{\mathbf{n}^{*}} \pi^{\xi}(g) d \xi, g \in G$. The equivalence is given by the Fourier transform over $N$, denoted by $\mathbf{F}$ :

$$
\mathbf{F} f(\xi, t)=(2 \pi)^{-n / 2} \int_{\mathbf{n}} f(\exp Y, t) e^{-i\langle\xi, Y\rangle} d Y, \quad f \in L^{2}(G) .
$$

This gives us an explicit decomposition of $\rho$ as a direct integral of induced representations from the normal subgroup $N$.

REMARK. $\pi^{\xi}$ need not be irreducible. For example if $G=\mathbb{R}^{2}$, thought of as $\mathbb{R} \cdot \mathbb{R}$, then $\pi^{\xi}$ acts on $L^{2}(\mathbb{R})$ while all the irreducible unitary representations of $G$ are characters. 
We will use primes to denote the objects on $\mathbf{n}^{*} \times \mathbb{R}$ corresponding to objects on $G$, i.e. functions by $u^{\prime}=u^{\prime}(\xi, t)$, operators by $A^{\prime}$. We will write $u_{\xi}(t)$ for the function of $t$ defined by $u_{\xi}=u^{\prime}(\xi, t)$. In particular for $L \in U(g), L=\mathbf{F}^{-1} \cdot L^{\prime} \cdot F$ where $L^{\prime}=\int_{\mathbf{n}^{*}} \pi^{\xi}(L) D \xi$. Thus $L \sim$ $\int_{\mathbf{n}^{*}} \pi^{\xi}(L) d \xi$.

Definition 2.1. A differential operator $L$ on a Lie group $G$ is semiglobally solvable if for every $f \in C_{c}^{\infty}(G)$, and for every relatively compact open set $U$ there exists $u \in C^{\infty}(G)$ such that $L u=f$ on $U$.

TheOREM 2.1. Let $L \in U(g)$. Suppose that there exists an $n$ variable polynomial $p$ such that for every $f \in C_{c}^{\infty}(G), \pi^{\xi}(L) u_{\xi}=f_{\xi}$ is solvable with $\xi \rightarrow p(\xi) u_{\xi}(t)$ a tempered distribution for a.e. $t \in \mathbb{R}$. Then $L$ is semiglobally solvable.

REMARK. This theorem is analogous to Theorem 2.1 in Lipsman [6]. The major differences are that we do not use the Plancherel Theorem and do not require invertibility of $\pi^{\xi}(L)$.

Proof of Theorem 2.1. Let $U$ be a relatively compact open set in $G$ and let $f \in C_{c}^{\infty}(G)$. It follows from the hypotheses of the theorem that there exist functions $u_{\xi}$ which satisfy $\pi^{\xi}(L) u_{\xi}=f_{\xi}$. Let $u^{\prime}(\xi, t)=u_{\xi}(t) . p(\xi)$ commutes with $L^{\prime}$ from which it follows that $L^{\prime} p(\xi) u^{\prime}(\xi, t)=p(\xi) L^{\prime} u^{\prime}(\xi, t)=p(\xi) f^{\prime}(\xi, t)$. Since $p(\xi) u^{\prime}(\xi, t)$ is tempered in $\xi$ for a.e. $t$ we can take its inverse Fourier transform. Let $u=$ $\mathbf{F}^{-1}\left(p \cdot u^{\prime}\right) . u$ is a distributional solution of $L u=p(D) f$ where $p(D)$ denotes the constant coefficient differential operator whose Fourier transform is $p(\xi)$.

From this point on the proof follows along the same lines as the proof of Theorem 2.1 in Lipsman [5]. For the sake of completeness we include this portion of the proof.

Let $h \in C_{c}^{\infty}(G)$. By the Dixmier Malliavin factorization ([2, Theorem 3.1]) we can factorize $h$ :

$$
h=\sum_{i=1}^{I} g_{i} * k_{i} \quad \text { where } g_{i}, k_{i} \in C_{c}^{\infty}(G), 1 \leq i \leq I .
$$

It follows from the global solvability of $p(D)[3,7]$ that there exist $\tilde{f}_{i} \in C^{\infty}(G)$ such that $p(D) \tilde{f}_{i}=k_{i}$. Let $\omega \in C_{c}^{\infty}(G)$ with $\omega \equiv 1$ on $U$ and let $f_{i}=\tilde{f}_{i} \cdot \omega$. Thus $f_{i} \in C_{c}^{\infty}(G), 1 \leq i \leq I$ and $p(D) f_{i}=k_{i}$ on $U$. 
By the above argument there exist distributions $u_{i}$ such that $L u_{i}=$ $p(D) f_{i}=k_{i}$ on $U$. Let

$$
u=\sum_{i=1}^{I} g_{i} * u_{i}
$$

$u \in C_{c}^{\infty}(G)$ since $g_{i} \in C_{c}^{\infty}(G)$. From the left invariance of $L$ it follows that

$$
L u=L\left(\sum_{i} g_{i} * u_{i}\right)=\sum_{i} g_{i} * L u_{i}=\sum_{i} g_{i} * k_{i}=h \quad \text { on } U .
$$

3. Elliptic and sub-elliptic 'operators. In the following two sections we apply Theorem 2.1 to prove solvability for certain specific classes of second order differential operators. In this section we investigate differential operators which are sums of squares of left invariant vector fields and sums of squares plus a linear term.

Under the representations $\pi^{\xi}$ which were discussed in $\S 2$ these operators are taken to Schrödinger operators. Thus a large proportion of what follows is analysis of Schrödinger operators with polynomial, trigonometric and exponential potentials.

Let $T \in \mathbf{s}, T \neq 0$. Let $\left\{X_{0}, \ldots, X_{n-1}\right\}$ be a basis for $\mathbf{n}$ with respect to which $\operatorname{ad}_{T}$ is in real Jordan form. It follows from (2.1) that

$$
\pi^{\xi}(T)=\frac{d}{d t}, \quad \pi^{\xi}\left(\mathbf{X}_{i}\right)=\left\langle\xi, e^{\operatorname{ad}_{t} T} X_{i}\right\rangle, \quad \xi \in \mathbf{n}^{*} .
$$

If $X_{i}$ corresponds to a generalized eigenspace with real eigenvalue $a$ then we have

$$
e^{\mathrm{ad}_{t T}} X_{i}=e^{a t} \sum_{j=0}^{i} \frac{t^{i-j}}{(i-j) !} X_{j}
$$

Thus

$$
\pi^{\xi}\left(X_{i}\right)=i e^{a t} \sum_{j=0}^{i} \frac{t^{i-j}}{(i-j) !} \xi_{j}
$$

Similarly for $X_{2 i}$ corresponding to a generalized eigenspace with complex eigenvalue $a+i b$ we have

$$
\pi^{\xi}\left(X_{2 i}\right)=i e^{a t} \sum_{j=0}^{i} \frac{t^{i-j}}{(i-j) !}\left(\xi_{2 j} \cos b t-\xi_{2 j+1} \sin b t\right) .
$$

$\left(\left(\xi_{2 j} \sin b t+\xi_{2 j+1} \cos b t\right)\right.$ for $\left.X_{2 i+1}\right)$. 
Let $L=-\left(T^{2}+\sum_{i \in I} X_{i}^{2}\right), I \subset\{0,1, \ldots,, n-1\}$. If follows that

$$
\pi^{\xi}(L)=-\frac{d^{2}}{d t^{2}}+\sum_{i \in I}\left\langle\xi, e^{\mathrm{ad}_{t i}} X_{i}\right\rangle^{2}=-\frac{d^{2}}{d t^{2}}+P_{\xi}(t)
$$

where $P_{\xi}(t)$ is the sum of non-negative terms of one of the following forms:

$$
e^{2 a t}\left(\sum_{k=0}^{K} \frac{t^{K-k}}{(K-k) !} \xi_{k}\right)^{2},
$$

$$
e^{2 a t}\left(\sum_{k=0}^{K} \frac{t^{K-k}}{(K-k) !}\left(\xi_{2 k} \cos b t-\xi_{2 k+1} \sin b t\right)\right)^{2} .
$$

Below are some examples of operators covered in this section.

EXAMPLe (1). $G$ is the " $a x+b$ " group: $S=\mathbb{R}, N=\mathbb{R}$ and the bracket of the two generators is $[T, X]=X$. With the global coordinates $g=(\exp x X) \cdot(\exp t T) \in G$ we have:

$$
L=\frac{\partial^{2}}{\partial t^{2}}-e^{2 t} \frac{\partial^{2}}{\partial x^{2}}, \quad L_{X}=-\frac{\partial^{2}}{\partial t^{2}}-e^{2 t} \frac{\partial^{2}}{\partial x^{2}}+e^{t} \frac{\partial}{\partial x} .
$$

Semiglobal solvability of these operators follows from Theorem 3.1.

EXAmple (2). $G$ is $N \cdot S$ where $N=\mathbb{R}^{2}, S=\mathbb{R}$ and the bracket relations are

$$
\left[T, X_{1}\right]=a X_{1}+b X_{2}, \quad\left[T, X_{2}\right]=-b X_{1}+a X_{2} .
$$

With the above coordinates we have for $L=-\left(T^{2}+X_{1}^{2}\right)$

$$
\begin{aligned}
L= & -\frac{\partial^{2}}{\partial t^{2}}-e^{2 a t}\left(\cos b t \frac{\partial}{\partial x_{1}}-\sin b t \frac{\partial}{\partial x_{2}}\right)^{2}, \\
L_{X_{2}}= & -\frac{\partial^{2}}{\partial t^{2}}-e^{2 a t}\left(\cos b t \frac{\partial}{\partial x_{1}}-\sin b t \frac{\partial}{\partial x_{2}}\right)^{2} \\
& +e^{a t}\left(\sin b t \frac{\partial}{\partial x_{1}}+\cos b t \frac{\partial}{\partial x_{2}}\right) .
\end{aligned}
$$

Again semiglobal solvability of these operators follows from Theorem 3.1.

EXAmPle (3). $G$ is the Mautner Group: $N=\mathbb{R}^{4}, S=\mathbb{R}$. The bracket relations between the generators are

$$
\begin{array}{ll}
{\left[T, X_{1}\right]=X_{2},} & {\left[T, X_{2}\right]=-X_{1},} \\
{\left[T, X_{3}\right]=\pi X_{4},} & {\left[T, X_{4}\right]=-\pi X_{3} .}
\end{array}
$$


With the above coordinates we have for $L=-\left(T^{2}+X_{1}^{2}+X_{3}^{2}\right)$,

$$
\begin{aligned}
L= & -\frac{\partial^{2}}{\partial t^{2}}-\left(\cos t \frac{\partial}{\partial x_{1}}-\sin t \frac{\partial^{2}}{\partial x_{2}}\right)^{2} \\
& +\left(\cos \pi t \frac{\partial}{\partial x_{3}}-\sin \pi t \frac{\partial}{\partial x_{4}}\right)^{2}, \\
L_{X_{2}}= & -\frac{\partial^{2}}{\partial t^{2}}-\left(\cos t \frac{\partial}{\partial x_{1}}-\sin t \frac{\partial}{\partial x_{2}}\right)^{2} \\
& +\left(\cos \pi t \frac{\partial}{\partial x_{3}}-\sin \pi t \frac{\partial}{\partial x_{4}}\right)^{2} \\
& +\left(\sin t \frac{\partial}{\partial x_{1}}+\cos t \frac{\partial}{\partial X_{2}}\right) .
\end{aligned}
$$

Semiglobal solvability for these operators follows from Theorem 3.1. This is an example of a non-type I group for which our approach works.

Before we state and prove the main results of this chapter we present some lemmas and propositions on Schrödinger operators which will be used in the proof of these results.

LEMMA 3.1. Let $V_{b}(t)=\left(\xi_{0} \cos b t+\xi_{1} \sin b t\right)^{2}, b \neq 0, \xi_{0}, \xi_{1} \in \mathbb{R}$. The zeros of $V_{b}$ are

$$
\left\{\frac{1}{b} \tan ^{-1}\left(-\xi_{0} / \xi_{1}\right)+\frac{\pi k}{b}: k \in \mathbb{Z}\right\}
$$

and

$$
V_{b}\left(\frac{1}{b} \tan ^{-1}\left(-\xi_{0} / \xi_{1}\right)+\frac{\pi k}{b}+t\right)=\left(\xi_{0}^{2}+\xi_{1}^{2}\right) \cdot \sin ^{2} b t .
$$

LEMMA 3.2. Let

$$
P(t)=\left[\sum_{k=0}^{K} \frac{t^{K-k}}{(K-k) !}\left(\xi_{2 k} \cos b t+\xi_{2 k+1} \sin b t\right)\right]^{2},
$$

$\xi_{0}, \ldots, \xi_{2 K+1} \in \mathbb{R}, b>0$. Given $0<\delta<\pi / b$ there exist $T>0, \varepsilon>0$ such that for $|t|>T$ and outside symmetric intervals of length $\delta$ around the points

$$
\left\{\frac{1}{b} \tan ^{-1}\left(-\xi_{0} / \xi_{1}\right)+\frac{\pi k}{b}: k \text { an integer }\right\}
$$

we have

$$
P(t) \geq \varepsilon\left(\xi_{0}^{2}+\xi_{1}^{2}\right)
$$


We omit the proofs of these lemmas which are elementary in nature. We note that Lemma 3.2 follows from Lemma 3.1 together with some straightforward estimates.

Proposition 3.1. Let $V: \mathbb{R}^{2} \rightarrow \mathbb{R}^{+} \cup\{0\}, V$ radial and $V \in L_{\text {loc }}^{2}\left(\mathbb{R}^{2}\right)$. Suppose there exist positive numbers $R>1, \rho, \lambda$ such that $[R, \infty)$ is a countable union of disjoint intervals, $I_{n}=\left[\alpha_{n}, \beta_{n}\right), n \in \mathbb{Z}$, with the following property:

For every positive integer $n$ there exists an interval $J_{n}, J_{n} \subset\left(\alpha_{n}+\rho\right.$, $\left.\beta_{n}-\rho\right)$, with $\left(l\left(J_{n}\right)+\rho\right) / \alpha_{n}<1$, such that $V(r) \geq \lambda$ for $r \in I_{n}-J_{n}$.

Let $A=-\Delta+V(r)$, where $\Delta$ represents the radial part of the Laplacian on $\mathbb{R}^{2}$. Define two weights on the plane by:

$$
\begin{aligned}
& \omega_{1}(r)= \begin{cases}1, & 0 \leq r \leq R, \\
r^{-2}, & r>R,\end{cases} \\
& \omega_{2}(r)= \begin{cases}1, & 0 \leq r \leq R, \\
r^{2}, & r>R .\end{cases}
\end{aligned}
$$

Then

$$
\|A \varphi\|_{\omega_{2}} \geq c(\lambda)\|\varphi\|_{\omega_{1}} \text { for all } \varphi \in C_{c}^{\infty}\left(\mathbb{R}^{2}\right), \varphi \text { radial }
$$

where $c(\lambda)=\min \left\{K_{1}, K_{2} \lambda\right\} ; K_{1}, K_{2}$ positive constants independent of $\lambda$. Furthermore we have the following inequality:

$$
\|\varphi\|_{\omega_{1}} \leq(1 / c(\lambda))(A \varphi, \varphi) \leq(1 / c(\lambda))\|A \varphi\|_{\omega_{2}} \cdot\|\omega\|_{\omega_{1}} .
$$

Proof. We will show that

$$
\|\varphi\|_{\omega_{1}}^{2} \leq(1 / c(\lambda))(A \varphi, \varphi)
$$

where $C(\lambda)=\min \left\{K_{1}, K_{2} \lambda\right\} ; K_{1}, K_{2}$ positive constants independent of $\lambda$. The proposition follows from this fact and the observation that

$$
(A \varphi, \varphi) \leq\|A \varphi\|_{\omega_{2}} \cdot\|\varphi\|_{\omega_{1}}
$$

which is a consequence of the Cauchy-Schwarz inequality.

Now we estimate $\|\varphi\|_{\omega_{1}}^{2}$ :

$$
\begin{aligned}
\|\varphi\|_{\omega_{1}}^{2} & =2 \pi \int_{0}^{\infty}|\varphi(r)|^{2} \omega_{1}(r) r d r \\
& =2 \pi \int_{0}^{R}|\varphi(r)|^{2} r d r+2 \pi \int_{R}^{\infty}|\varphi(r)|^{2} r^{-1} d r .
\end{aligned}
$$

First we estimate $2 \pi \int_{0}^{R}|\varphi(r)|^{2} r d r$. Let $g \in C_{c}^{\infty}\left(\mathbb{R}^{2}\right), g$ radial satisfying $g(r) \leq 1, g(r)=1$ for $r \in[0, R]$ and $g(r)=0$ for $r>R+\rho$. 
Let $M=\max \left|g^{\prime}(r)\right|^{2}, Q=\operatorname{diam}(\operatorname{supp} g)$. It follows from the uncertainty principle that $\|\psi\| \leq \operatorname{diam}(\operatorname{supp} \psi) \cdot\|\nabla \psi\|_{L^{2}\left(\mathbb{R}^{2}, \mathbb{C}^{2}\right)}$. If $\psi$ is radial it follows that

$$
\|\psi\| \leq \operatorname{diam}(\operatorname{supp} \psi) \cdot\left\|\psi^{\prime}(r)\right\| .
$$

An application of this inequality to $\varphi(r) \cdot g(r)$ gives us

$$
\begin{aligned}
2 \pi \int_{0}^{R}|\varphi(r)|^{2} r d r \leq & 4 \pi Q^{2} \int_{0}^{\infty}\left|\varphi^{\prime}(r)\right|^{2} r d r \\
& +4 \pi Q^{2} M \int_{R}^{R+\rho}|\varphi(r)|^{2} r d r .
\end{aligned}
$$

Using this together with the fact that $V(r) \geq \lambda$ for $r \in[R, R+\rho]$ we obtain

$$
2 \pi \int_{0}^{R}|\varphi(r)|^{2} r d r \leq \max \left\{2 Q^{2}, \frac{2 Q^{2} M}{\lambda}\right\} \cdot(A \varphi, \varphi) .
$$

Now we estimate $\int_{R}^{\infty}|\varphi(r)|^{2} r^{-1} d r$ :

$$
\int_{R}^{\infty}|\varphi(r)|^{2} r^{-1} d r=\sum_{n=1}^{\infty} \int_{I_{n}}|\varphi(r)|^{2} r^{-1} d r .
$$

Let $\left\{g_{n}\right\} \subset C_{c}^{\infty}\left(\mathbb{R}^{2}\right), g_{n}$ radial and satisfying $g_{n}(r) \leq 1, g_{n}(r)=1$ for $r \in J_{n}, g_{n}(r)=0$ for $r \notin J_{n}+(-\rho / 2, \rho / 2)$ and $\left|\left(g_{n}^{\prime}(r)\right)\right|^{2} \leq M, M$ independent of $n$.

$$
\int_{I_{n}}|\varphi(r)|^{2} r^{-1} d r=\int_{I_{n}-J_{n}}|\varphi(r)|^{2} r^{-1} d r+\int_{J_{n}}|\varphi(r)|^{2} r^{-1} d r .
$$

The potential $V(r)$ is bounded away from zero on $I_{n}-J_{n}$. Thus

$$
\int_{I_{n}-J_{n}}|\varphi(r)|^{2} r^{-1} d r \leq \frac{1}{\lambda \cdot R^{2}} \int_{I_{n}} V(r) \cdot|\varphi(r)|^{2} r d r .
$$

An application of the Poincaré inequality to $\varphi(r) \cdot g_{n}(r) \cdot r^{-1 / 2}$ yields

$$
\begin{aligned}
\int_{J_{n}}|\varphi(r)|^{2} r^{-1} d r \leq & \left(l\left(J_{n}\right)+\rho\right)^{2} \int_{I_{n}}\left|\left(\varphi(r) \cdot g_{n}(r)\right)^{\prime}\right|^{2} r^{-1} d r \\
& +\frac{1}{4} \int_{I_{n}}|\varphi(r)|^{2} \cdot\left(g_{n}(r)\right)^{2} r^{-1} d r
\end{aligned}
$$

From this it follows that

$$
\begin{aligned}
& \text { (3.10) } \frac{3}{4} \int_{J_{n}}|\varphi(r)|^{2} r^{-1} d r \\
& \quad \leq \max \left\{2, \frac{2 M+1 / 4}{\lambda}\right\} \int_{I_{n}}\left(\left|\varphi^{\prime}(r)\right|^{2}+V(r) \cdot|\varphi(r)|^{2}\right) r d r .
\end{aligned}
$$


It follows from (3.9) and (3.10) that

$$
\begin{aligned}
\int_{I_{n}}|\varphi(r)|^{2} r^{-1} d r & \\
& \leq \max \left\{\frac{1}{\lambda R^{2}}, \frac{8}{3}, \frac{8 M+1}{3 \lambda}\right\} \int_{I_{n}}\left(\left|\varphi^{\prime}(r)\right|^{2}+V(r) \cdot|\varphi(r)|^{2}\right) r d r .
\end{aligned}
$$

Thus

$$
\begin{aligned}
& 2 \pi \int_{R}^{\infty}|\varphi(r)|^{2} r^{-1} d r \\
&=2 \pi \sum_{n=1}^{\infty} \int_{I_{n}}|\varphi(r)|^{2} r^{-1} d r \\
& \leq 2 \pi \cdot \max \left\{\frac{8}{3}, \frac{8 m+1+3 / R^{2}}{3 \lambda}\right\} \\
& \times \int_{0}^{\infty}\left(\left|\varphi^{\prime}(r)\right|^{2}+V(r) \cdot|\varphi(r)|^{2}\right) r d r \\
&= \max \left\{\frac{8}{3}, \frac{8 m+1+3 R^{2}}{3 \lambda}\right\}(A \varphi, \varphi) .
\end{aligned}
$$

From (3.8), (3.11) it follows that

$$
(A \varphi, \varphi) \geq c(\lambda)\|\varphi\|_{\omega_{1}}^{2}
$$

where $c(\lambda)=\min \left\{K_{1}, K_{2} \lambda\right\} ; K_{1}, K_{2}$ positive constants independent of $\lambda$.

Proposition 3.2. Let $V: \mathbb{R} \rightarrow \mathbb{R}^{+} \cup\{0\}, V \in L_{\text {loc }}^{2}(\mathbb{R})$. Suppose there exist positive numbers $T, \rho, \lambda, m_{1}, m_{2}$ such that $\mathbb{R}-[-T, T)$ is a countable union of disjoint intervals $I_{n}, I_{n}=\left[\alpha_{n}, \beta_{n}\right), n \in \mathbb{Z}^{+}$, $m_{1}<l\left(I_{n}\right)<m_{2}$, with the following property: For every $n \in \mathbb{Z}^{+}$there exists an interval $J_{n}$,

$$
J_{n} \subset\left[\alpha_{n}+\rho, \beta_{n}-\rho\right] \text { and } V(t) \geq \lambda \text { for } t \in I_{n}-J_{n} .
$$

Then

$$
\left(\left(-\frac{d^{2}}{d t^{2}}+V(t)\right) \varphi, \varphi\right)_{L^{2}(\mathbb{R})} \geq c(\lambda)\|\varphi\|^{2} \text { for all } \varphi \in C_{c}^{\infty}(\mathbb{R})
$$

where $c(\lambda)=\min \left\{K_{1}, K_{2}\right\}, K_{1}, K_{2}$ positive constants independently of $\lambda$.

REMARK. This proposition is a generalization of Theorem 3.3 in [6] and therefore we omit the proof. In our proposition the potential, $V(t)$, it not necessarily bounded away from zero for large $|t|$. The 
proposition roughly says that it suffices to have the potential bounded away from zero "part of the time" in order to get the desired inequality.

TheOREM 3.1. $G=N \cdot S, N \approx \mathbb{R}^{n}, S \approx \mathbb{R}, N$ normal in $G$. Let $T \in \mathbf{s}, T \neq 0$. Let $\left\{X_{0}, \ldots, X_{n-1}\right\}$ be a basis for $\mathbf{n}$ with respect to which $\operatorname{ad}_{T}$ is in real Jordan form. Let

$$
\begin{aligned}
L & =-\left(T^{2}+\sum_{i \in I} X_{i}^{2}\right), \quad I \subset\{0, \ldots, n-1\}, \\
L_{X} & =L+X, \quad X \in \mathbf{n} .
\end{aligned}
$$

Then $L, L_{X}$ are semiglobally solvable.

Proof of Theorem 3.1. We prove the theorem only for the case when one of the $X_{i}$ 's appearing in $L$ corresponds to an eigenvalue of $\operatorname{ad}_{T}$ with non-zero real part. When no such $X_{i}$ exists the proof is similar to the proof of Theorem 5.4 in [6]. The major difference is that here our Schrödinger operators have potentials which are bounded away from zero "part of the time" and Proposition 3.2 must be used instead of the analogous proposition in [6].

Assume that the real part of the eigenvalue with non-zero real part is positive. The proof for the other case is similar. Denote $\min _{1 \leq j \leq J^{\prime \prime}}\left\{a_{j}\right\}$ by $a$.

The potential $P_{\xi}$ can be written as $P_{\xi}=P_{\xi}^{+}+P_{\xi}^{-}$where $P_{\xi}^{+}$consists of terms of the form (3.4) or (3.5) with $a>0$ and $P_{\xi}^{-}$of terms with $a \leq 0$. In the sequel a positive constant, $\alpha$, appears. If $P_{\xi}^{-}=0$ then $\alpha$ can be chosen to be equal to $a$ (smallest positive real part). Otherwise $\alpha$ is chosen to be less than or equal to $a$. More will be said about $\alpha$ at the point in the proof where its choice becomes significant.

REMARK. Invertibility of the $\pi_{\xi}(L)$ 's on $L^{2}(\mathbb{R})$ would easily lead to a definition for $u_{\xi}$. That this invertibility is not the case in general is illustrated by the Laplacian of the " $a x+b$ " group. If $L$ is the Laplacian on the " $a x+b$ " group then

$$
\pi^{\xi}(L)=-\frac{d^{2}}{d t^{2}}+\xi^{2} e^{2 t}
$$

and this operator is not boundedly invertible on $L^{2}(\mathbb{R})$ since the potential $\xi^{2} e^{2 t}$ goes to zero at $-\infty$. In fact this operator doesn't have a bounded right inverse on $L^{2}\left(\mathbb{R}, e^{2 t} d t\right)$ even though on this weighted Hilbert space $\pi^{\xi}(L)$ satisfies $\left\|\pi^{\xi}(L) \varphi\right\| \geq C \cdot\|\varphi\|$ for $\varphi \in C_{c}^{\infty}(\mathbb{R})$. The 
problem is that $\pi^{\xi}(L)\left(C_{c}^{\infty}(\mathbb{R})\right)$ is not dense in $L^{2}\left(\mathbb{R}, e^{2 t} d t\right)$ and thus its closure is not onto.

Thus to prove the existence of these $u_{\xi}$ 's we utilize the following change of variables:

$$
r=e^{\alpha t}, \quad 0<\alpha \leq a .
$$

If $h$ is a function in $t$ let $\tilde{h}$ denote the corresponding function in $r$. $\tilde{h}(r)=h\left(\frac{1}{\alpha} \log r\right)$. Under this change of variables the operator $\pi^{\xi}(L)$ is transformed into $\tilde{\pi}^{\xi}(L)$ where

$$
\tilde{\pi}^{\xi}(L)=\alpha^{2} r^{2}\left(\left(-\frac{d^{2}}{d r^{2}}-\frac{1}{r} \frac{d}{d r}\right)+Q_{\xi}(r)\right) .
$$

Here $\alpha^{2} r^{2} Q_{\xi}(r)=\tilde{P}_{\xi}(r) ; P_{\xi}(t)$ the potential in $\pi^{\xi}(L)$.

We think of functions in $r$ as radial functions on the plane. If $\Delta$ represents the radial part of the Laplacian in 2 variables then $\tilde{\pi}^{\xi}(L)=$ $\alpha^{2} r^{2}\left(-\Delta+Q_{\xi}\right)$. Our aim is to find a function $u_{\xi}$ such that

$$
\tilde{\pi}(L) \tilde{u}_{\xi}=\tilde{f}_{\xi}
$$

Since $f_{\xi} \in C_{c}^{\infty}(\mathbb{R})$ we have $\tilde{f}_{\xi} \in C_{0}^{\infty}\left(\mathbb{R}^{2}-\{0\}\right)$. Thus $(\alpha r)^{-2} \tilde{f}_{\xi} \in$ $C_{c}^{\infty}\left(\mathbb{R}^{2}-\{0\}\right)$. If $A_{\xi}=-\Delta+Q_{\xi}$ then our problem is equivalent to finding a sufficiently nice function $\tilde{u}_{\xi}$ such that

$$
A_{\xi} \tilde{u}_{\xi}=(\alpha r)^{-2} \tilde{f}_{\xi}
$$

For some of the operators covered by the theorem (including the Laplacian on the " $a x+b$ " group) $A_{\xi}$ is invertible as an operator on $L^{2}\left(\mathbb{R}^{2}\right)$. Unfortunately most of the operators give rise to $A_{\xi}$ 's which are not invertible on $L^{2}\left(\mathbb{R}^{2}\right)$. We shall see that $A_{\xi}$ is invertible as an operator from $L^{2}\left(\mathbb{R}^{2}, \omega_{1}\right)$ to $L^{2}\left(\mathbb{R}^{2}, \omega_{2}\right)$ with appropriate domain where $\omega_{1}, \omega_{2}$ are appropriate weights on the plane. (We are thinking of radial functions only as we will be doing in the sequel unless explicitly stated otherwise.)

When we make the change of variables we get $\tilde{P}_{\xi}(r)=\alpha^{2} r^{2} Q_{\xi}(r)$. It follows that we can write $Q_{\xi}=Q_{\xi}^{+}+Q_{\xi}^{-}$where $Q_{\xi}^{+}, Q_{\xi}^{-}$are sums of terms of the following forms with $a>0, a \leq 0$ respectively:

$$
\frac{1}{\alpha^{2}} r^{2(a / \alpha-1)}\left(\sum_{k=0}^{K} \frac{\left(\alpha^{-1} \log r\right)^{K-k}}{(K-k) !} \xi_{k}\right)^{2},
$$




$$
\frac{1}{\alpha^{2}} r^{2(a / \alpha-1)}\left(\sum_{k=0}^{K} \frac{\left(\alpha^{-1} \log r\right)^{K-K}}{(K-k) !}\right.
$$

$$
\left.\cdot\left(\xi_{2 k} \cos (b / \alpha) \log r-\xi_{2 k+1} \sin (b / \alpha) \log r\right)\right)^{2}
$$

We need to analyze potentials of these forms. First we analyze a potential of form (3.5') with $a \geq \alpha$ which we denote by $q_{\xi}(r)$.

By Lemma 3.2, given $0<\delta<\pi /(b / \alpha)$, there exist $T>0, \varepsilon>0$ such that for $t>T, q_{\xi}\left(e^{a t}\right) \geq \alpha^{-2} \varepsilon\left(\xi_{0}^{2}+\xi_{1}^{2}\right)$ as long as $t$ is outside symmetric intervals of length $\delta$ around the zeros of

$$
V_{b / \alpha}(t)=\left(\xi_{0} \cos (b / \alpha) t-\xi_{1} \sin (b / \alpha) t\right)^{2} .
$$

Let

$$
t_{0}=\tan ^{-1}\left(\xi_{0} / \xi_{1}\right) \in\left[-\frac{\pi}{2}, \frac{\pi}{2}\right] .
$$

All the zeros of $V_{b / \alpha}$ are of the form

$$
\frac{t_{0}}{(b / \alpha)}+\frac{\pi n}{(b / \alpha)}, \quad n \in \mathbb{Z}
$$

Let

$$
\begin{aligned}
& I_{n}^{\prime}=\left[\frac{t_{0}}{(b / \alpha)}+\frac{\pi}{(b / \alpha)}(n-1 / 2), \frac{t_{0}}{(b / \alpha)}+\frac{\pi}{(b / \alpha)}(n+1 / 2)\right], \\
& J_{n}^{\prime}=\left[\frac{t_{0}}{(b / \alpha)}+\frac{\pi n}{(b / \alpha)}-\delta / 2, \frac{t_{0}}{(b / \alpha)}+\frac{\pi n}{(b / \alpha)}+\delta / 2\right] .
\end{aligned}
$$

(The $J_{n}^{\prime}$ 's are the symmetric intervals indicated above.) Changing variables we can conclude that $q_{\xi}(r) \geq \alpha^{-2} \varepsilon\left(\xi_{0}^{2}+\xi_{2}^{2}\right)$ for $r>e^{T}$ and outside the corresponding intervals $J_{n}$ (for $r$ in $I_{n}-J_{n}$.) It follows that

$$
l\left(J_{n}\right)=e^{t_{0} /(b / a)+\pi n /(b / a)}\left(e^{\delta / 2}-e^{-\delta / 2}\right) .
$$

We can choose $\delta$ small enough so that there exists $\rho>0$ for which

$$
\frac{l\left(J_{n}\right)+\rho}{e^{t_{0} /(b / a)+(\pi /(b / a))(n-1 / 2)}}<1
$$

independent of $n \in \mathbb{Z}$. (The denominator in the above expression is the value of the left endpoint of $I_{n}$.) Let $R=e^{T}$. It follows from Proposition 3.1 that

$$
\left\|\left(-\Delta+q_{\xi}(r)\right) \varphi\right\|_{\omega_{2}} \geq c\left(\xi_{0}, \xi_{1}\right) \cdot\|\varphi\|_{\omega_{1}}
$$

for $\varphi \in C_{c}^{\infty}\left(\mathbb{R}^{2}\right)$ where $C\left(\xi_{0}, \xi_{1}\right)=\alpha^{-2} \min \left\{K_{1}, K_{2}\left(\xi_{0}^{2}+\xi_{1}^{2}\right)\right\} ; K_{1}$, $K_{2}$ positive constants independent of $\xi$, and $-\omega_{1}, \omega_{2}$ weights on the plane, as described in the statement of Proposition 3.1. 
We now analyze a potential of the form $\left(3.4^{\prime}\right)$ with $a \geq \alpha$ which we denote by $q_{\xi}(r)$. For large enough $R, q_{\xi}(r) \geq \xi_{0} / \alpha^{2}$ as long as $r>R$. It follows from Proposition 3.1 that

$$
\left\|\left(-\Delta+q_{\xi}(r)\right) \varphi\right\|_{\omega_{2}} \geq c\left(\xi_{0}\right) \cdot\|\varphi\|_{\omega_{1}}
$$

for $\varphi \in C_{c}^{\infty}\left(\mathbb{R}^{2}\right)$ where $c\left(\xi_{0}\right)=\alpha^{-2} \min \left\{K_{1}, K_{2} \xi_{0}^{2}\right\}, K_{1}, K_{2}$ positive constants independent of $\xi$.

This analysis shows that if we consider $-\Delta+Q_{\xi}$ as an operator from $L^{2}\left(\mathbb{R}^{2}, \omega_{1}\right)$ to $L^{2}\left(\mathbb{R}^{2}, \omega_{2}\right)$ with domain equal to $C_{0}^{\infty}\left(\mathbb{R}^{2}\right)$, where the $R$ in the definitions of $\omega_{1}, \omega_{2}$ is chosen to be the largest $R$ corresponding to the terms in $Q_{\xi}^{+}$then

$$
\left\|\left(-\Delta+Q_{\xi}\right) \varphi\right\|_{\omega_{1}} \leq c(\xi) \cdot\|\varphi\|_{\omega_{2}}
$$

where $c(\xi)$ is a constant of the form appearing in (3.12) or (3.13) depending on whether $Q_{\xi}$ contains terms of the form $\left(3.4^{\prime}\right)$ and/or $\left(3.5^{\prime}\right)$.

In the remainder of the proof we must differentiate between 2 cases, $P_{\xi}^{-}=0$ and $P_{\xi}^{-} \neq 0$. The difference between the 2 cases is that in the first case it makes sense to choose the domain of $A_{\xi}$ as $C_{c}^{\infty}\left(\mathbb{R}^{2}\right)$ while in the second case we must make do with $C_{c}^{\infty}\left(\mathbb{R}^{2}-\{0\}\right)$. In both cases we prove that $A_{\xi}$ is invertible from $L^{2}\left(\mathbb{R}^{2}, \omega_{1}\right)$ to $L^{2}\left(\mathbb{R}^{2}, \omega_{2}\right)$. More precisely we have :

LEMMA 3.3. Think of $A_{\xi}=-\Delta+Q_{\xi}$ as a linear operator from $L^{2}\left(\mathbb{R}^{2}, \omega_{1}\right)$ to $L^{2}\left(\mathbb{R}^{2}, \omega_{2}\right)$ with domain as described above. Then $\bar{A}_{\xi}$ is onto and has a bounded right inverse satisfying $\left\|\bar{A}_{\xi}^{-1}\right\| \leq 1 /(c(\xi))$ where $c(\xi)$ is the constant appearing in (3.14).

Proof of of Lemma 3.3. We first remark that $A_{\xi}$ is closable since $A_{\xi}^{*}$ is densely defined. (All functions of the form $\psi_{1} / \omega_{2}, \psi_{1} \in C_{c}^{\infty}\left(\mathbb{R}^{2}-\{0\}\right)$, are contained in $D\left(\mathscr{A}_{\xi}^{*}\right)$.)

It is a direct consequence of inequality (3.14) and Proposition 3.1 that

$$
\begin{aligned}
\|\varphi\|_{\omega_{1}}^{2} & \leq 1 /(c(\xi))\left(\left(-\Delta+Q_{\xi}^{+}\right) \varphi, \varphi\right) \leq 1 /(c(\xi))\left(A_{\xi} \varphi, \varphi\right) \\
& \leq 1 /(c(\xi)) \cdot\left\|A_{\xi} \varphi\right\|_{\omega_{2}} \cdot\|\varphi\|_{\omega_{1}} .
\end{aligned}
$$

Thus $\left\|A_{\xi} \varphi\right\|_{\omega_{2}} \geq c(\xi) \cdot\|\varphi\|_{\omega_{1}}$ for $\varphi \in D\left(A_{\xi}\right)$ where $c(\xi)$ is as in (3.18). To finish the proof of this lemma it suffices to show that the range of $A_{\xi}$ is dense in $L^{2}\left(\mathbb{R}^{2}, \omega_{2}\right)$. Here we must differentiate between the 2 
cases:

$P_{\xi}^{-}=0$ : Thus $Q_{\xi}^{-}=0, D\left(A_{\xi}\right)=C_{c}^{\infty}\left(\mathbb{R}^{2}\right)$. Suppose $\psi \in A_{\xi}\left(D\left(A_{\xi}\right)\right)^{\perp}$, i.e., for all $\varphi \in C_{c}^{\infty}\left(\mathbb{R}^{2}\right), \varphi$ radial, $\left(A_{\xi} \varphi, \psi\right)_{\omega_{2}}=0$. We must show that $\psi=0$. Define

$$
\psi_{1}(r)= \begin{cases}\psi(r), & 0 \leq r \leq R, \\ r^{2} \psi(r), & r>R .\end{cases}
$$

A bootstrapping type argument using Sobolev's lemma (see [9], Lemma preceding Theorem IX.24 and Theorem IX.24) shows that $\psi_{1} \in$ $C^{\infty}\left(\mathbb{R}^{2}-\{0\}\right)$ and that $\psi_{1} \in C_{0}\left(\mathbb{R}^{2}\right)$. In addition it is easy to check that $\psi_{1} \in L^{2}\left(\mathbb{R}^{2}, \omega_{1}\right)$.

Make the change of variables $r=e^{\alpha t}$. Let $u(t)=\psi_{1}\left(e^{\alpha t}\right)$. Under this change of variables $\alpha^{2} r^{2} \Delta$ becomes $\left(d^{2} / d t^{2}\right) . u \in C^{\infty}(\mathbb{R})$ since $\psi_{1} \in C^{\infty}\left(\mathbb{R}^{2}-\{0\}\right)$, and satisfies

$$
\frac{d^{2} u}{d t^{2}}=P_{\xi} \cdot u
$$

Again it is easy to check that $u \in L^{2}\left(\mathbb{R}, \omega_{3}\right)$ where

$$
\omega_{3}(r)= \begin{cases}e^{\alpha t}, & t<\log R, \\ 1, & t \geq \log R .\end{cases}
$$

Suppose $u \neq 0$. W.L.O.G. $u\left(t_{0}\right)>0$ for some $t_{0}$. Since $u \neq 0$ and is continuous it must be non-zero at a point where $P_{\xi}(t)$ does not vanish. Thus we can assume that $P_{\xi}\left(t_{0}\right)>0$. It follows from (3.15) that $u^{\prime \prime}\left(t_{0}\right)>0$. If $u^{\prime}\left(t_{0}\right) \geq 0$ then $u$ is a non-decreasing function on $\left[t_{0}, \infty\right)$ and can't be in $L^{2}\left(\mathbb{R}, \omega_{3}\right)$. Thus $u^{\prime}\left(t_{0}\right)<0$ and $u$ is a decreasing function on $\left(-\infty, t_{0}\right]$ with $\lim _{t \rightarrow-\infty} u(t)=\infty$. This leads to a contradiction: Since $\psi_{1} \in C^{0}\left(\mathbb{R}^{2}\right), u$ must have a limit at $-\infty$. $\left(\psi_{1}\right.$ is continuous at the origin.) Therefore $u=0$ from which it follows that $\psi_{1}=0$, which in turn implies that $\psi=0$. This completes the proof of density of $A_{\xi}\left(C_{c}^{\infty}\left(\mathbb{R}^{2}\right)\right)$ in $L^{2}\left(\mathbb{R}^{2}, \omega_{2}\right)$ for the case when $P_{\xi}^{-}=0$.

$P_{\xi}^{-} \neq 0$ : In this case $D\left(A_{\xi}\right)=C_{0}^{\infty}\left(\mathbb{R}^{2}-\{0\}\right)$. Suppose $\psi \in$ $A_{\xi}\left(D\left(A_{\xi}\right)\right)^{\perp}$, i.e. for all $\varphi \in C_{0}^{\infty}\left(\mathbb{R}^{2}-\{0\}\right)$ we have $\left(A_{\xi} \varphi, \psi\right)_{\omega_{2}}=0$. We must show that $\psi=0$. As in the case where $P_{\xi}^{-}=0$ we have $\psi_{1} \in C^{\infty}\left(\mathbb{R}^{2}-\{0\}\right)$.

We again make the change of variables $r=e^{\alpha t}$. Let $u(t)=\psi_{1}\left(e^{\alpha t}\right)$. $u \in L^{2}\left(\mathbb{R}^{2}, \omega_{3}\right) \cap C^{\infty}(\mathbb{R})$ and satisfies

$$
\frac{d^{2} u}{d t^{2}}=P_{\xi} \cdot u
$$


Suppose $u \neq 0$. W.L.O.G $u\left(t_{0}\right)>0$ for some $t_{0}$. As before we can assume that $P_{\xi}\left(t_{0}\right)>0$. Thus $u^{\prime \prime}\left(t_{0}\right)>0$ by (3.16).

If $u^{\prime}\left(t_{0}\right) \geq 0$ then $u$ is a non-decreasing function on $\left[t_{0}, \infty\right)$ and can't be in $L^{2}\left(\mathbb{R}, \omega_{3}\right)$. Thus $u^{\prime}\left(t_{0}\right)<0$ from which it follows that $u$ is a decreasing function on $\left(-\infty, t_{0}\right]$. We will show that for small enough $\alpha, u$ is not square integrable against $e^{\alpha t}$ at $-\infty$ and this will be a contradiction to $u$ being in $L^{2}\left(\mathbb{R}, \omega_{3}\right)$.

$P_{\xi}(t)$ contains a term of the form (3.4) or (3.5) where $a \leq 0$. We will analyze the case where we have a term of the form (3.5). The other case is similar. Thus

$$
P_{\xi}(t) \geq e^{2 a t}\left(\sum_{k=0}^{K} \frac{t^{K-k}}{(K-k) !}\left(\xi_{2 k} \cos b t-\xi_{2 k+1} \sin b t\right)\right)^{2}, \quad a \leq 0 .
$$

By Lemma 3.2 given $\delta>0$ there exist $T>0, \varepsilon>0$ such that $P_{\xi}(t) \geq$ $\varepsilon\left(\xi_{0}^{2}+\xi_{1}^{2}\right)$ as long as $t<-T$ and is outside symmetric intervals of length $\delta$ around the zeros of $V_{b}(t)=\left(\xi_{0} \cos b t+\xi_{1} \sin b t\right)^{2}$. We can assume that $t_{0}$ is outside these intervals and that $t_{0}<-T$. ( $u$ is decreasing on $\left(-\infty, t_{0}\right]$.) Let $\gamma>0$ be small enough so that $t_{0}-\gamma$ stays inside the "good" interval. By the Taylor formula with remainder we have

$$
\begin{aligned}
u\left(t_{0}-\gamma\right)=u\left(t_{0}\right)-\gamma u^{\prime}\left(t_{0}\right)+\frac{\gamma^{2}}{2} u^{\prime \prime}(s) \geq u\left(t_{0}\right)+\frac{\gamma^{2}}{2} u^{\prime \prime}(s), & \left(u^{\prime}\left(t_{0}\right)<0\right) \text { for some } s, t_{0}-\gamma \leq s \leq t_{0} .
\end{aligned}
$$

By (3.16) we have

$$
\begin{aligned}
u\left(t_{0}-\gamma\right) & \geq u\left(t_{0}\right)+\frac{\gamma^{2}}{2} P_{\xi}(s) u(s) \\
& \geq u\left(t_{0}\right)+\frac{\gamma^{2}}{2} \cdot \varepsilon \cdot\left(\xi_{0}^{2}+\xi_{1}^{2}\right) u\left(t_{0}\right),
\end{aligned}
$$

$(s$ is in a "good" interval).

Let $\kappa=\left(\gamma^{2} / 2\right) \cdot \varepsilon \cdot\left(\xi_{0}^{2}+\xi_{1}^{2}\right)$. Then

$$
u\left(t_{0}-\gamma\right) \geq u\left(t_{0}\right)(1+\kappa) .
$$

Replacing $t_{0}$ by $t_{0}-\pi / b\left(\pi / b\right.$ is the period of $\left.V_{b}\right)$ we get

$$
\begin{aligned}
u\left(t_{0}-\frac{\pi}{b}-\gamma\right) \geq u\left(t_{0}-\frac{\pi}{b}\right)(1+\kappa) \geq u\left(t_{0}\right)(1+\kappa)^{2} . \\
\left(u\left(t_{0}-\frac{\pi}{b}\right) \geq u\left(t_{0}-\gamma\right) \text { since } u \text { is decreasing }\right) .
\end{aligned}
$$

By induction it follows that for $n \in \mathbb{Z}^{+}$,

$$
\begin{aligned}
u\left(t_{0}-\frac{\pi n}{b}-\gamma\right) & \geq u\left(t_{0}\right)(1+\kappa)^{n+1} \\
& =u\left(t_{0}\right)(1+\kappa)^{b / \pi^{(n+1) \pi / b}} .
\end{aligned}
$$


If $\alpha$ is chosen so that $e^{\alpha}<(1+\kappa)^{b / \pi}$ then it follows that $u$ is not square integrable against $e^{\alpha t}$ at $-\infty$. Thus $u=0$ from which it follows that $\psi_{1}=0$, which in turn implies that $\psi=0$. This completes the proof of the density of $A_{\xi}\left(C_{c}^{\infty}\left(\mathbb{R}^{2}, \omega_{2}\right)\right)$ in $L^{2}\left(\mathbb{R}^{2}, \omega_{2}\right)$ for the case when $P_{\xi}^{-} \neq 0$, and concludes the proof of Lemma 3.3.

Now we can define $\tilde{u}_{\xi}$ to be

$$
\tilde{u}_{\xi}=\bar{A}_{\xi}^{-1}\left(\frac{1}{(\alpha r)^{2}} \tilde{f}_{\xi}\right)
$$

$\bar{A}_{\xi}$ acts as $-\Delta+Q_{\xi}$ in the punctured plane. Thus

$$
\bar{A}_{\xi} \tilde{u}_{\xi}=\left(-\Delta+Q_{\xi}\right) \tilde{u}_{\xi}=\frac{1}{(\alpha r)^{2}} \cdot \tilde{f}_{\xi}
$$

This implies that

$$
\Delta \tilde{u}_{\xi}=F(r)
$$

where

$$
F(r) \equiv Q_{\xi}(r) \cdot \tilde{u}_{\xi}-\frac{1}{(\alpha r)^{2}} \tilde{f}_{\xi}
$$

is in $L_{\text {loc }}^{2}\left(\mathbb{R}^{2}-\{0\}\right)$ and $\Delta$ is the distributional Laplacian. By a bootstrapping type argument similar to the one mentioned above $\tilde{u}_{\xi} \in$ $C^{\infty}\left(\mathbb{R}^{2}-\{0\}\right)$.

We define $u_{\xi}=\tilde{u}_{\xi}\left(e^{\alpha t}\right)$. It follows that $\pi^{\xi}(L) u_{\xi}=f_{\xi}$.

$$
\tilde{u}_{\xi} \in L^{2}\left(\mathbb{R}^{2}, \omega_{1}\right) \Rightarrow u_{\xi} \in L^{2}\left(\mathbb{R}^{2}, \omega_{3}\right) .
$$

Below we estimate $\left\|u_{\xi}\right\|_{\omega_{3}}$ :

$$
\begin{aligned}
\left\|u_{\xi}\right\|_{\omega_{3}} & =\left\|\tilde{u}_{\xi}\right\|_{\omega_{1}}=\left\|\bar{A}_{\xi}^{-1}\left((\alpha r)^{-2} \tilde{f}_{\xi}\right)\right\|_{\omega_{1}} \\
& \leq\left\|\bar{A}_{\xi}^{-1}\right\|_{\omega_{1}} \cdot\left\|(\alpha r)^{-1} \tilde{f}\right\|_{\omega_{2}} \leq(1 / c(\xi)) \cdot\left\|(\alpha r)^{-2} \tilde{f}_{\xi}\right\|_{\omega_{2}} \\
& =\left(1 /\left(\alpha^{2} c(\xi)\right)\right) \cdot\left\|r^{-2} \cdot \tilde{f}_{\xi}\right\|_{\omega_{2}}
\end{aligned}
$$

where $c(\xi)$ is as in (3.18). Thus $\alpha^{2} c(\xi)$ is of the form

$$
\min \left\{K_{1}, K_{2}\left(\xi_{0}^{2}+\xi_{1}^{2}\right)\right\}
$$

if the potential contains a term of the form (3.5); and of the form $\min \left\{K_{1}, K_{2} \xi_{0}^{2}\right\}$ if the potential contains a term of the form (3.4). (The potential might contain terms of both types in which case either form of $c(\xi)$ is sufficient in the following argument.) In the remainder of the proof we will assume that $\alpha^{2} c(\xi)$ is of the form $\min \left\{K_{1}, K_{2}\left(\xi_{0}^{2}+\xi_{1}^{2}\right)\right\}$; the other case can be dealt with similarly. 
Let $p(\xi)=\xi_{0}^{2}+\xi_{1}^{2}$. In order to apply Theorem 2.1 we need to show that $\xi \rightarrow p(\xi) u_{\xi}(t)$ is a tempered distribution for a.e. $t$. It follows from the definition of $p$ that $p(\xi) /\left(\alpha^{2} c(\xi)\right)$ is bounded by some $K$. As supp $f_{\xi}$ is independent of $\xi$ it follows that $\left\|\left(1 / r^{2}\right) \tilde{f}_{\xi}\right\|_{\omega_{3}} \leq L\left\|f_{\xi}\right\|$ for some constant $L$. The following calculation demonstrates that $\xi \rightarrow p(\xi) u_{\xi}(t)$ is in $L^{2}\left(\mathbb{R}^{2}\right)$ for a.e. $t$ :

$$
\begin{aligned}
& \int_{\mathbb{R}} \int_{\mathbb{R}^{n}}\left|u_{\xi}(t)\right|^{2}(p(\xi))^{2} d \xi \omega_{3}(t) d t \\
& \quad=\int_{\mathbb{R}^{n}} \int_{\mathbb{R}}\left|u_{\xi}(t)\right|^{2} \omega_{3}(t) d t p(\xi)^{2} d \xi \\
& \quad \leq \int_{\mathbb{R}^{n}} p(\xi)^{2}\left(1 / \alpha^{4} c(\xi)^{2}\right)\left\|\frac{1}{r^{2}} \tilde{f}_{\xi}\right\|_{\omega_{3}}^{2} d \xi \\
& \quad \leq K^{2} L^{2} \int_{\mathbb{R}^{n}}\left\|f_{\xi}\right\|^{2}<\infty .
\end{aligned}
$$

Thus $\xi \rightarrow p(\xi) u_{\xi}(t)$ is tempered in $\xi$ for a.e. $t$. We can now apply Theorem 2.1 to obtain semiglobal solvability of $L$.

The proof of semiglobal solvability for $L_{X}$ follows along the same lines. The operator $\tilde{\pi}^{\xi}\left(L_{X}\right)$ can be written as $\alpha^{2} r^{2} H_{\xi}$ where $H_{\xi}=$ $A_{\xi}+B_{\xi}$ where $A_{\xi}$ is as before and $B_{\xi}=\left(1 /(\alpha r)^{2}\right) \tilde{\pi}^{\xi}(X)$.

We want to thing of $H_{\xi}$ as an operator from $L^{2}\left(\mathbb{R}^{2}, \omega_{1}\right)$ to $L^{2}\left(\mathbb{R}^{2}, \omega_{2}\right)$ with $D\left(H_{\xi}\right)=C_{c}^{\infty}\left(\mathbb{R}^{2}-\{0\}\right)$. The following calculation shows that $H_{\xi}$ satisfies the same type of inequality as $A_{\xi}((3.14))$ : It follows from Proposition 3.1 that for $\varphi \in D\left(H_{\xi}\right)$

$$
\begin{aligned}
\|\varphi\|_{\omega_{1}}^{2} & \leq(1 / c(\xi)) \cdot\left(A_{\xi} \varphi, \varphi\right) \\
& \leq(1 / c(\xi)) \cdot \sqrt{\left(A_{\xi} \varphi, \varphi\right)^{2}+\left(i B_{\xi} \varphi, \varphi\right)^{2}} \\
& \quad\left(B_{\xi}\right. \text { is skew-symmetric) } \\
& =(1 / c(\xi)) \cdot\left|\left(H_{\xi} \varphi, \varphi\right)\right| \leq(1 / c(\xi)) \cdot\left\|H_{\xi} \varphi\right\|_{\omega_{2}} \cdot\|\varphi\|_{\omega_{1}} .
\end{aligned}
$$

Thus

$$
\left\|H_{\xi} \varphi\right\|_{\omega_{2}} \geq c(\xi) \cdot\|\varphi\|_{\omega_{1}}
$$

where $c(\xi)$ is as in (3.14).

In order to show that $\bar{H}_{\xi}$ has a bounded right inverse it remains to demonstrate that $R_{g}\left(H_{\xi}\right)$ is dense in $L^{2}\left(\mathbb{R}^{2}, \omega_{2}\right)$ as before. This is much simpler than before and follows from the skew-symmetry of $B_{\xi}$. The remainder of the proof of global solvability for $L_{X}$ is similar to the proof of $L$.

REMARK. Theorem 3.1 discusses operators of the form $L+X, L$ a sum of squares. Similar results hold for operators of the form $L+\lambda X$, 
if $\operatorname{Re}(\lambda) \neq 0$ and $X \in Z(\mathbf{g})$. For in this case $\pi^{\xi}(L+\lambda X)=\pi^{\xi}(L)+$ $\lambda \pi^{\xi}(X)$ where $\pi^{\xi}(X) \in \mathbb{C}$. It follows that $\pi^{\xi}(L+\lambda X)$ is invertible on $L^{2}(\mathbb{R})$ with inverse bounded in terms of $\operatorname{Re}(\lambda)$. Semiglobal solvability follows from a straightforward application of Theorem 2.1 (see [4]).

The case $\operatorname{Re}(\lambda)=0$ is less tractable. Many of the operators discussed here are strictly positive on $L^{2}(\mathbb{R})$. Unfortunately the dependence of the lower bound of the spectrum on the representation parameter is not linear except in special cases (e.g. the Heisenberg group.) For example it follows from [4, pg. 144] that

$$
-\frac{d}{d t^{2}}+\left(\frac{t^{2}}{2} \xi_{2}+t \xi_{1}+\xi_{2}\right)^{2}
$$

has lowest eigenvalue of order $\xi_{2}^{2 / 3}$. This seems to suggest adding on, not a linear term, but a term with order balancing that of $L$. We hope to consider such operators in future work.

4. Parabolic operators. In this chapter we give another application of Theorem 2.1. Here the operators in question are parabolic. In the notation of the previous chapters these are operators of the form $L=T-\sum_{i \in I} X_{i}^{2}$. Under the representation $\pi^{\xi}$ these operators are taken to first order ordinary differential operators. We will use integrating factors to solve the resulting differential equations. Below are examples in this chapter:

EXAMPLES. (1) $G$ is the " $a x+b$ " group. With the global coordinates $g=(\exp x X) \cdot(\exp t T) \in G$ we have

$$
L=\frac{\partial}{\partial t}-e^{2 t} \frac{\partial^{2}}{\partial x^{2}}
$$

Semiglobal solvability of $L$ follows from Theorem 4.1 .

(2) $G$ is $N \cdot S$ where $N=\mathbb{R}^{2}, S=\mathbb{R}$ and the brackets are

$$
\left[T, X_{1}\right]=a X_{1}+b X_{2}, \quad\left[T, X_{2}\right]=-b X_{1}+a X_{2} .
$$

Let $L_{1}=T-X_{1}^{2}, L_{2}=T-X_{1}^{2}-X_{2}^{2}$. With the above coordinates we have

$$
\begin{aligned}
L_{1}= & \frac{\partial}{\partial t}-e^{2 a t}\left(\cos b t \frac{\partial}{\partial x_{1}}+\sin b t \frac{\partial}{\partial x_{2}}\right)^{2} \\
L_{2}= & \frac{\partial}{\partial t}-e^{2 a t}\left(\cos b t \frac{\partial}{\partial x_{1}}+\sin b t \frac{\partial}{\partial x_{2}}\right)^{2} \\
& -e^{a t}\left(-\sin b t \frac{\partial}{\partial x_{1}}+\cos b t \frac{\partial}{\partial x_{2}}\right)^{2}
\end{aligned}
$$


Semiglobal solvability of these operators follows from Theorem 4.1.

TheOREM 4.1. Let $G=N \cdot S, N \approx \mathbb{R}^{n}, S \approx \mathbb{R}, N$ normal in $G$. Let $T \in \mathbf{s}, T \neq 0$. Let $\left\{X_{0}, \ldots, X_{n-1}\right\}$ be a basis for $\mathbf{n}$ with respect to which $\mathrm{ad}_{T}$ is in real Jordan form. Let

$$
L=T-\sum_{i \in I} X_{i}^{2}, \quad I \subset\{0, \ldots, n-1\} .
$$

Then $L$ is semiglobally solvable.

Proof of Theorem 4.1. Let $f \in C_{c}^{\infty}(G)$. Thus supp $f_{\xi} \subseteq[c, d]$ independent of $\xi$.

It follows from (2.1) that $\pi^{\xi}(L)=d / d t+P_{\xi}(t)$ where $P_{\xi}$ is the sum of terms of the form (3.4), (3.5). Let $q_{\xi}$ be a typical term of the form (3.5). (Terms of the form (3.4) can be dealt with similarly.)

$\pi^{\xi}(L)$ is a first order differential operator. We can solve

$$
\pi^{\xi}(L) u_{\xi}=f_{\xi}
$$

with the aid of the integrating factor

$$
I_{\xi}(t)=e^{\int_{c}^{t} P_{\xi}(s) d s} .
$$

A solution to $(4.1)$ is

$$
u_{\xi}(t)=\left(I_{\xi}(t)\right)^{-1} \int_{c}^{t} I_{\xi}(s) f_{\xi}(s) d s .
$$

Let $p(\xi)=\xi_{0}^{2}+\xi_{1}^{2}$. We will show that $\xi \rightarrow p(\xi) u_{\xi}(t)$ is tempered in $\xi$ for a.e. $t$. Semiglobal solvability of $L$ will then follow from Theorem 2.1.

First we estimate $\left\|u_{\xi}(t) e^{a t}\right\|_{2}^{2}$ where $a$ is the same $a$ appearing in $q_{\xi}(t)$. ( $a$ might be 0 .)

$$
\begin{aligned}
& \int_{\mathbf{R}}\left|u_{\xi}(t)\right|^{2} e^{2 a t} d t=\int_{c}^{\infty}\left|u_{\xi}(t)\right|^{2} e^{2 a t} d t \\
& =\int_{c}^{d}\left|u_{\xi}(t)\right|^{2} e^{2 a t} d t+\int_{d}^{\infty}\left|u_{\xi}(t)\right|^{2} e^{2 a t} d t
\end{aligned}
$$

It follows from the Cauchy-Schwarz inequality that

$$
\begin{aligned}
(4.3) \int_{c}^{d} & \left|u_{\xi}(t)\right|^{2} e^{2 a t} d t=\int_{c}^{d}\left(I_{\xi}(t)\right)^{-2}\left|\int_{c}^{t} I_{\xi}(s) f_{\xi}(s) d s\right|^{2} e^{2 a t} d t \\
& \leq \int_{c}^{d}\left(I_{\xi}(t)\right)^{-2}\left(\int_{c}^{t}\left(I_{\xi}(s)\right)^{2} d s\right) \cdot\left(\int_{c}^{t}\left|f_{\xi}(s)\right|^{2} d s\right) e^{2 a t} d t \\
& \leq e^{2 a d}(d-c)^{2}\left\|f_{\xi}\right\|_{2}^{2}
\end{aligned}
$$


where in the last inequality we have used that $I_{\xi}(t)$ is an increasing function.

It follows from the analysis done in $\S 3$ that there exists $\varepsilon>0$, $m>0, \gamma>0$ for which $[d, \infty)$ can be written as the disjoint union of intervals $J_{n}=\left[a_{n}, b_{n}\right), I_{n}=\left[b_{n}, a_{n+1}\right)$ such that $l\left(I_{n}\right)=\gamma l\left(J_{n}\right)$, $m \geq \max \left\{l\left(I_{n}\right), l\left(J_{n}\right)\right\}$, and such that

$$
P_{\xi}(t) \geq q_{\xi}(t) \geq \varepsilon e^{2 a t}\left(\xi_{0}^{2}+\xi_{1}^{2}\right)
$$

on $J_{n}$. (We can always adjust $d$ so that it will match up with a left endpoint of a "good" interval.) Since $\operatorname{supp} f_{\xi} \subseteq[c, d]$ we have

$$
\begin{aligned}
& \int_{d}^{\infty}\left|u_{\xi}(t)\right|^{2} e^{2 a t} d t \\
& \quad=\int_{d}^{\infty}\left(I_{\xi}(t)\right)^{-2} e^{2 a t} d t \cdot\left|\int_{c}^{d} I_{\xi}(s) f_{\xi}(s) d s\right|^{2} \cdot \\
& \int_{d}^{\infty}\left(I_{\xi}(t)\right)^{-2} e^{2 a t} d t \\
& \quad=\sum_{n=1}^{\infty} \int_{J_{n}}\left(I_{\xi}(t)\right)^{-2} e^{2 a t} d t+\sum_{n=1}^{\infty} \int_{I_{n}}\left(I_{\xi}(t)\right)^{-2} e^{2 a t} d t \\
& \quad \leq\left(1+e^{m} \gamma\right) \sum_{n=1}^{\infty} \int_{J_{n}}\left(I_{\xi}(t)\right)^{-2} e^{2 a t} d t \\
& \quad=K \cdot \sum_{n=1}^{\infty} \int_{J_{n}}\left(I_{\xi}(t)\right)^{-2} e^{2 a t} d t
\end{aligned}
$$

since $I_{\xi}$ is an increasing function.

$$
\begin{aligned}
\int_{J_{n}}\left(I_{\xi}(t)\right)^{-2} e^{2 a t} d t & \leq \frac{1}{2 \varepsilon\left(\xi_{0}^{2}+\xi_{1}^{2}\right)} \int_{J_{n}} e^{-2 \int_{c}^{t} P_{\xi}(s) d s} 2 P_{\xi}(t) d t \\
& =\frac{1}{2 \varepsilon\left(\xi_{0}^{2}+\xi_{1}^{2}\right)}\left[\left(I_{\xi}\left(a_{n}\right)\right)^{-2}-\left(I_{\xi}\left(b_{n}\right)\right)^{-2}\right]
\end{aligned}
$$

Thus

$$
\begin{aligned}
& \sum_{n=1}^{N} \int_{J_{n}}\left(I_{\xi}(t)\right)^{-2} e^{-2 a t} d t \\
& \quad \leq \frac{1}{2 \varepsilon\left(\xi_{0}^{2}+\xi_{1}^{2}\right)} \sum_{n=1}^{N}\left[\left(I_{\xi}\left(a_{n}\right)\right)^{-2}-\left(I_{\xi}\left(b_{n}\right)\right)^{-2}\right]
\end{aligned}
$$


where we again have used that $I_{\xi}$ is increasing. Since $I_{\xi}(t) \rightarrow \infty$ as $f \rightarrow \infty$ we can conclude that

$$
\int_{d}^{\infty}\left(I_{\xi}(t)\right)^{-2} e^{2 a t} \leq \frac{k}{2 \varepsilon\left(\xi_{0}^{2}+\xi_{1}^{2}\right)}\left(I_{\xi}(d)\right)^{-2}
$$

Using this estimate in (4.4) we get

$$
\begin{aligned}
\int_{d}^{\infty}\left|u_{\xi}(t)\right|^{2} e^{2 a t} d t & \leq \frac{K}{2 \varepsilon\left(\xi_{0}^{2}+\xi_{1}^{2}\right)}\left(I_{\xi}(d)\right)^{-2}\left(I_{\xi}(d)\right)^{2}(d-c)\left\|f_{\xi}\right\|_{2}^{2} \\
& =\frac{K}{2 \varepsilon\left(\xi_{0}^{2}+\xi_{1}^{2}\right)}(d-c)\left\|f_{\xi}\right\|_{2}^{2}
\end{aligned}
$$

Estimates (4.3), (4.5) together give

$$
\int_{\mathbf{R}}\left|u_{\xi}(t)\right|^{2} e^{2 a t} d t \leq K_{1}\left\|f_{\xi}\right\|_{2}^{2}+\frac{K_{2}}{\left(\xi_{0}^{2}+\xi_{1}^{2}\right)}\left\|f_{\xi}\right\|_{2}^{2}
$$

for some constants $K_{1}, K_{2}$. From (4.6) and Fubini's Theorem it follows that

$$
\begin{aligned}
\int_{\mathbf{R}} \int_{\mathbb{R}^{n}} & \left|u_{\xi}(t)\right|^{2}\left(\xi_{0}^{2}+\xi_{1}^{2}\right)^{2} d \xi e^{2 a t} d t \\
\quad & \int_{\mathbf{R}^{n}} \int_{\mathbf{R}}\left|u_{\xi}(t)\right|^{2} e^{2 a t} d t\left(\xi_{0}^{2}+\xi_{1}^{2}\right)^{2} d \xi \\
& \leq \int_{\mathbf{R}^{n}}\left[K_{1}\left\|f_{\xi}\right\|_{2}^{2}+\frac{K_{2}}{\left(\xi_{0}^{2}+\xi_{1}^{2}\right)}\left\|f_{\xi}\right\|_{2}^{2}\right]\left(\xi_{0}^{2}+\xi_{1}^{2}\right)^{2} d \xi \\
& \leq \int_{\mathbf{R}^{n}}\left[K_{1}\left\|\left(\xi_{0}^{2}+\xi_{1}^{2}\right) f_{\xi}\right\|_{2}^{2}+K_{2}\left(\xi_{0}^{2}+\xi_{1}^{2}\right)\left\|f_{\xi}\right\|_{2}^{2}\right] d \xi \\
& \leq \int_{\mathbf{R}^{n}}\left[K_{1}\left\|\left(\xi_{0}^{2}+\xi_{1}^{2}\right) f_{\xi}\right\|_{2}^{2}+K_{2}\left\|\left(\xi_{0}^{2}+\xi_{1}^{2}\right) f_{\xi}\right\|_{2}^{2}+K_{2}\left\|f_{\xi}\right\|_{2}^{2}\right] d \xi \\
& \leq\left(K_{1}+K_{2}\right) \int_{\mathbb{R}^{n}}\left\|\pi^{\xi}\left(X_{0}^{2}+X_{2}^{2}\right) f_{\xi}\right\|_{2}^{2} d \xi+K_{2} \cdot \int_{\mathbb{R}^{n}}\left\|f_{\xi}\right\|_{2}^{2} d \xi \\
& =\left(K_{1}+K_{2}\right)\left\|\left(X_{0}^{2}+X_{1}^{2}\right) f\right\|_{2}^{2}+K_{2}\|f\|_{2}^{2}<\infty
\end{aligned}
$$

where we have used the Plancherel Theorem and that $f \in C_{c}^{\infty}(G)$. Hence $\xi \rightarrow p(\xi) u_{\xi}(t)$ is in $L^{2}\left(\mathbb{R}^{n}\right)$ for a.e. $t$. It now follows from Theorem 2.1 that $L$ is semiglobally solvable.

REMARK. A recent paper of Levy-Bruhl dealing with solvability questions has been brought to my attention [5]. In this paper LevyBruhl proves solvability results for many of the same classes of operators that have been treated here. His approach differs from ours in that he does not use representation theory. 


\section{REFERENCES}

[1] L. Corwin, A representation-theoretic criterion for local solvability of left invariant differential operators on nilpotent Lie groups, Trans. Amer. Math. Soc., 264 (1981), 113-120.

[2] J. Dixmier and P. Malliavin, Factorisations de Fonctions et de Vecteurs, Bull. Sci. Math., 102 (1978), 305-330.

[3] L. Ehrenpreis, Solution of some problems of division, Amer. J. Math., 76 (1954), 883-903.

[4] C. Fefferman, The Uncertainty Principle, Bull. Amer. Math. Soc., 9, no. 2 (1983), 129-206.

[5] P. Levy-Bruhl, Remarques sur la resolubilité d'équations différentielles, a propos de résultats de R. L. Lipsman, Comm. Partial Differential Equations, 13 (1988), 769-773.

[6] R. Lipsman, Solvability of invariant differential operators with variable coeffcients, Comm. Partial Differential Equations, 10 (1985), 1261-1361.

[7] _ Sobolev criteria for solvability of invariant differential operators, Comm. Partial Differential Equations, 12 (1987), 327-349.

[8] B. Malgrange, Existence et approximation des solutions des équations aux derivées partielles et des équations de convolution, Ann. Inst. Fourier, 6 (1955), 271-355.

[9] M. Reed and B. Simon, Fourier Analysis, Self-Adjointness, Academic Press, Orlando, 1975.

[10] C. Rockland, Hypoellipticity on the Heisenberg Group-representation-theoretic criteria, Trans. Amer. Math. Soc., 240 (1978), 1-52.

[11] L. Rothschild, Local solvability of left invariant differential operators on the Heisenberg group, Proc. Amer. Math. Soc., 74 (1979), 383-388.

Received March 18, 1988 and in revised form August 25, 1988.

State University of New York at Albany

AlbaNY, NY 12222 


\section{PACIFIC JOURNAL OF MATHEMATICS EDITORS}

\author{
V. S. VARADARAJAN \\ (Managing Editor) \\ University of California \\ Los Angeles, CA 90024-1555-05 \\ Herbert Clemens \\ University of Utah \\ Salt Lake City, UT 84112 \\ ThOMAS ENRIGHT \\ University of California, San Diego \\ La Jolla, CA 92093
}

\section{R. FINN}

Stanford University

Stanford, CA 94305

HeRmann FlaschKa

University of Arizona

Tucson, AZ 85721

VAUGHAN F. R. JONES

University of California

Berkeley, CA 94720

Steven KerckhofF

Stanford University

Stanford, CA 94305

\author{
RobION KIRBY \\ University of California \\ Berkeley, CA 94720 \\ C. C. Moore \\ University of California \\ Berkeley, CA 94720 \\ HAROLD STARK \\ University of California, San Diego \\ La Jolla, CA 92093
}

\section{ASSOCIATE EDITORS}
R. ARENS
E. F. BECKenBach
B. H. NeumanN
F. WOLF
K. YoshidA (1906-1982)
(1904-1989)

\section{SUPPORTING INSTITUTIONS}

UNIVERSITY OF ARIZONA

UNIVERSITY OF BRITISH COLUMBIA

CALIFORNIA INSTITUTE OF TECHNOLOGY

UNIVERSITY OF CALIFORNIA

MONTANA STATE UNIVERSITY

UNIVERSITY OF NEVADA, RENO

NEW MEXICO STATE UNIVERSITY

OREGON STATE UNIVERSITY
UNIVERSITY OF OREGON

UNIVERSITY OF SOUTHERN CALIFORNIA

STANFORD UNIVERSITY

UNIVERSITY OF HAWAII

UNIVERSITY OF TOKYO

UNIVERSITY OF UTAH

WASHINGTON STATE UNIVERSITY

UNIVERSITY OF WASHINGTON 


\section{Pacific Journal of Mathematics}

Vol. 142, No. $1 \quad$ January, 1990

Marco Andreatta, Mauro Beltrametti and Andrew Sommese, Generic properties of the adjuction mapping for singular surfaces and applications

Chen-Lian Chuang and Pjek-Hwee Lee, On regular subdirect products of

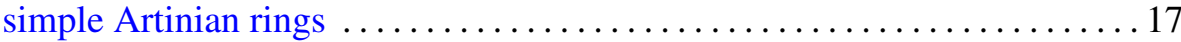

Fernando Giménez and Vicente Miquel Molina, Volume estimates for real hypersurfaces of a Kaehler manifold with strictly positive holomorphic sectional and antiholomorphic Ricci curvatures $\ldots \ldots \ldots \ldots \ldots \ldots 23$

Richard J. Griego and Andrzej Korzeniowski, Asymptotics for certain Wiener integrals associated with higher order differential operators

Abdeslam Mesnaoui, Unitary bordism of classifying spaces of quaternion

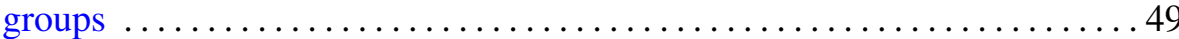

Abdeslam Mesnaoui, Unitary cobordism of classifying spaces of quaternion

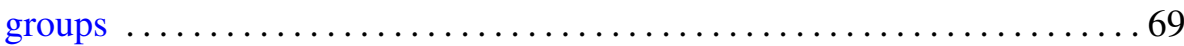

Jesper M. Møller, On equivariant function spaces $\ldots \ldots \ldots \ldots \ldots \ldots \ldots \ldots$

Bassam Nassrallah, A $q$-analogue of Appell's $F_{1}$ function, its integral representation and transformations

Peter A Ohring, Solvability of invariant differential operators on metabelian

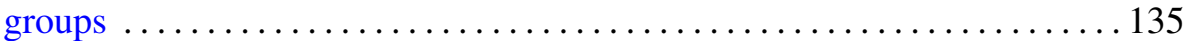

Athanase Papadopoulos and R. C. Penner, Enumerating pseudo-Anosov foliations

Ti-Jun Xiao and Liang Jin, On complete second order linear differential

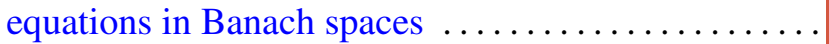

Carl Widland and Robert F. Lax, Weierstrass points on Gorenstein curves 